\title{
Ultrasound-guided allograft pancreas biopsy: Safety and diagnostic use
}

Fan H, Turner SS, Yeung K, Moser S, Cook T, Roufosse C, Charif R, McLean A, Taube D, Tait P, Muthusamy AR

\section{Background}

- Post transplant monitoring of enteric drained pancreas transplants is largely limited to serum glucose and amylase

- In response to abnormal amylase and/or glucose results, patients often receive empirical immunotherapy

- Pancreas allograft biopsy is seldom performed because of concerns regarding reliability and safety

- Our aim was to analyse our experience of performing allograft pancreas biopsies to determine their usefulness and complication rates

\section{Methods}

- All pancreas allograft biopsies performed at our centre from 2009-2016 were included

- Retrospective study of several electronic databases and MDT outcomes focussing on biopsy procedure, adequacy, histology, post-procedure amylase and haemoglobin, any reported complications and management.

\section{Results}

- 91 transplants were performed during the study period (76 SPK, I 4 PAK, I PTA)

- SPKs received Campath/Tac/MMF

- PAKs received Tac/MMF

- $\quad$ PTA received IL2RA/Tac/MMF

- 72 biopsies were performed in 42 patients

- 7I were Ultrasound-guided, I was CT-guided

- A 16 or 18 gauge needle was used

- 67 were 'indication’ biopsies. See Figure I.

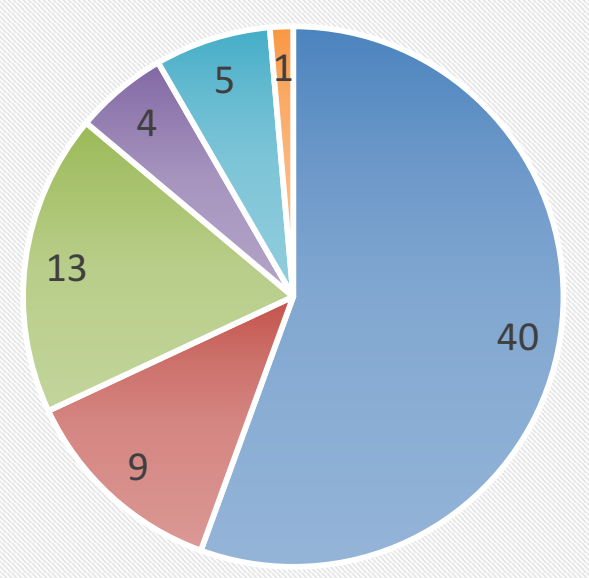

\section{Elevated amylase \\ - Deranged glucose \\ - Both \\ - Follow-up \\ - Surveillance \\ Low C-peptide and insulin}

Figure I. Indications for pancreas allograft biopsy. $86 \%$ were for elevated amylase, deranged glucose or both.

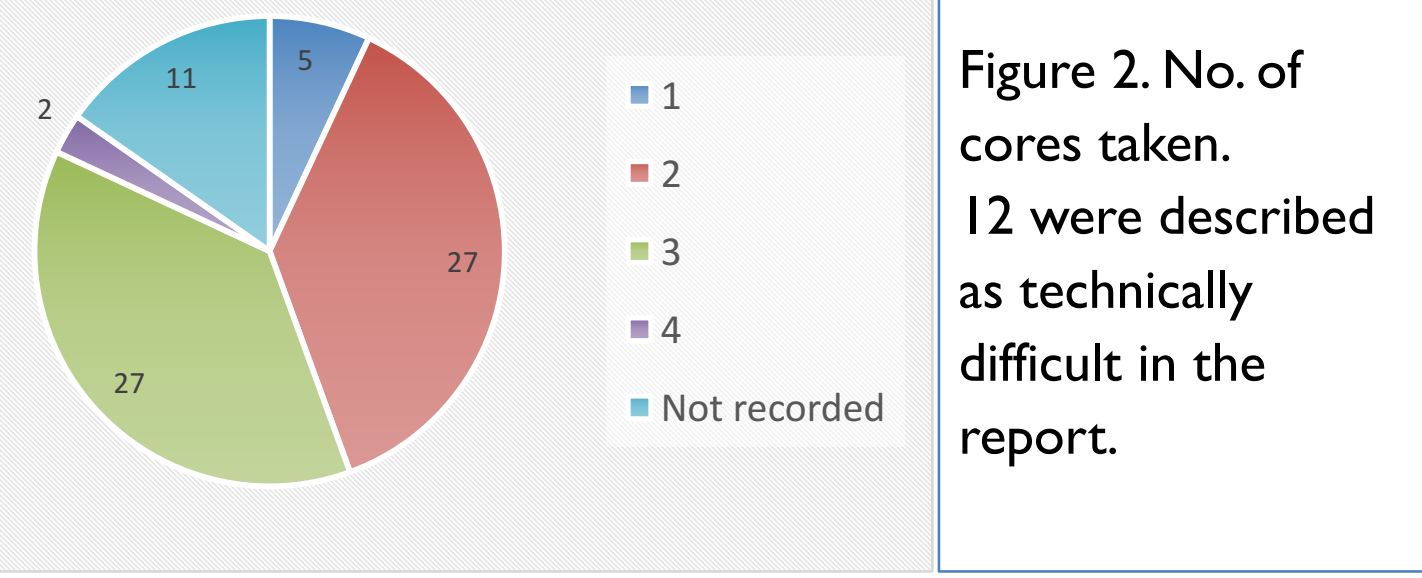

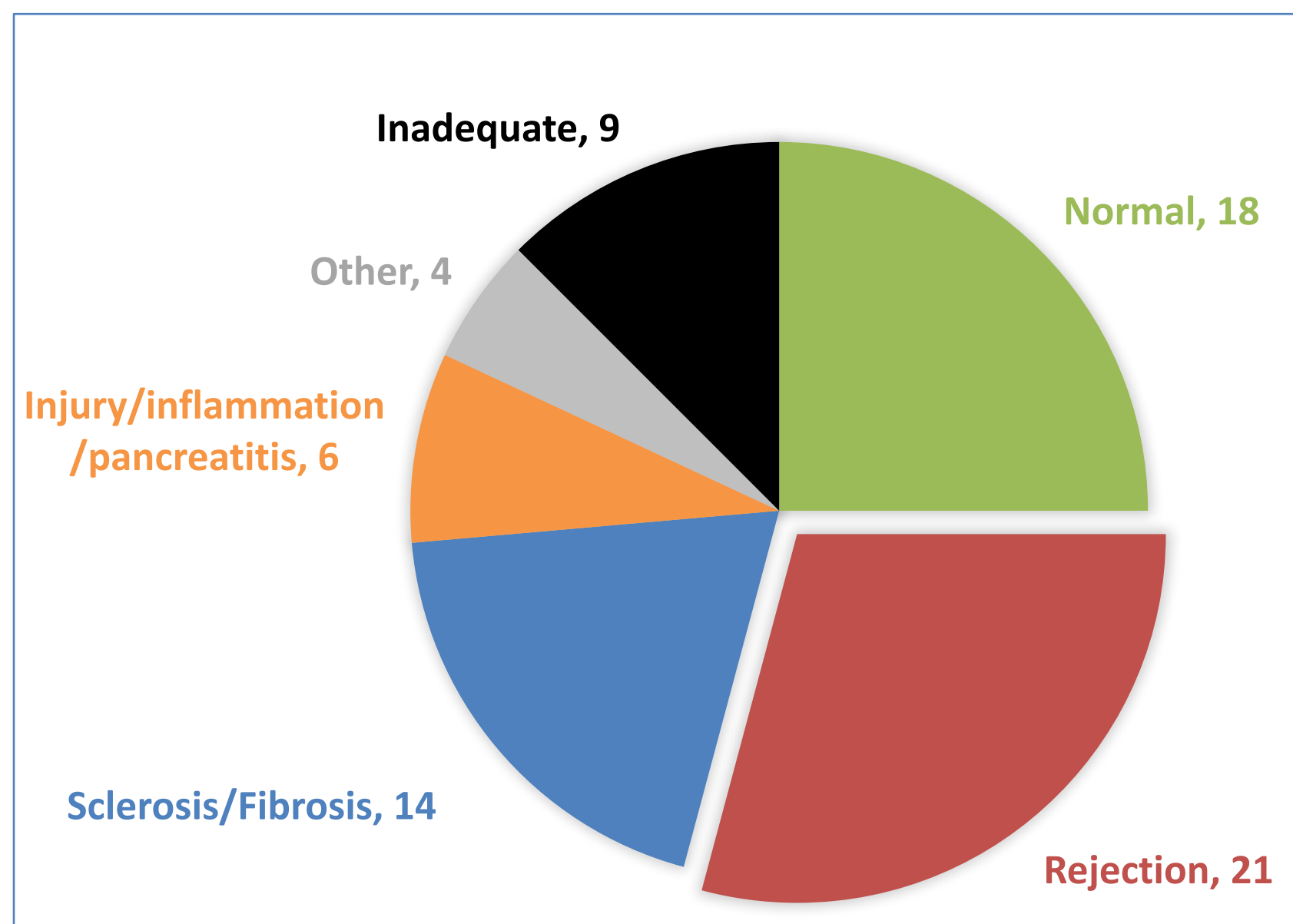

Figure 3. Histology of all 72 biopsies. Mild to moderate septal expansion was counted as normal. 20 of the rejections were T-cell mediated; one of these has additional features suggesting antibody-mediated rejection (AMR), the final one was mild AMR. 'Other' included I with granulomas, I arteriolar hyalinosis and 2 ischaemia.

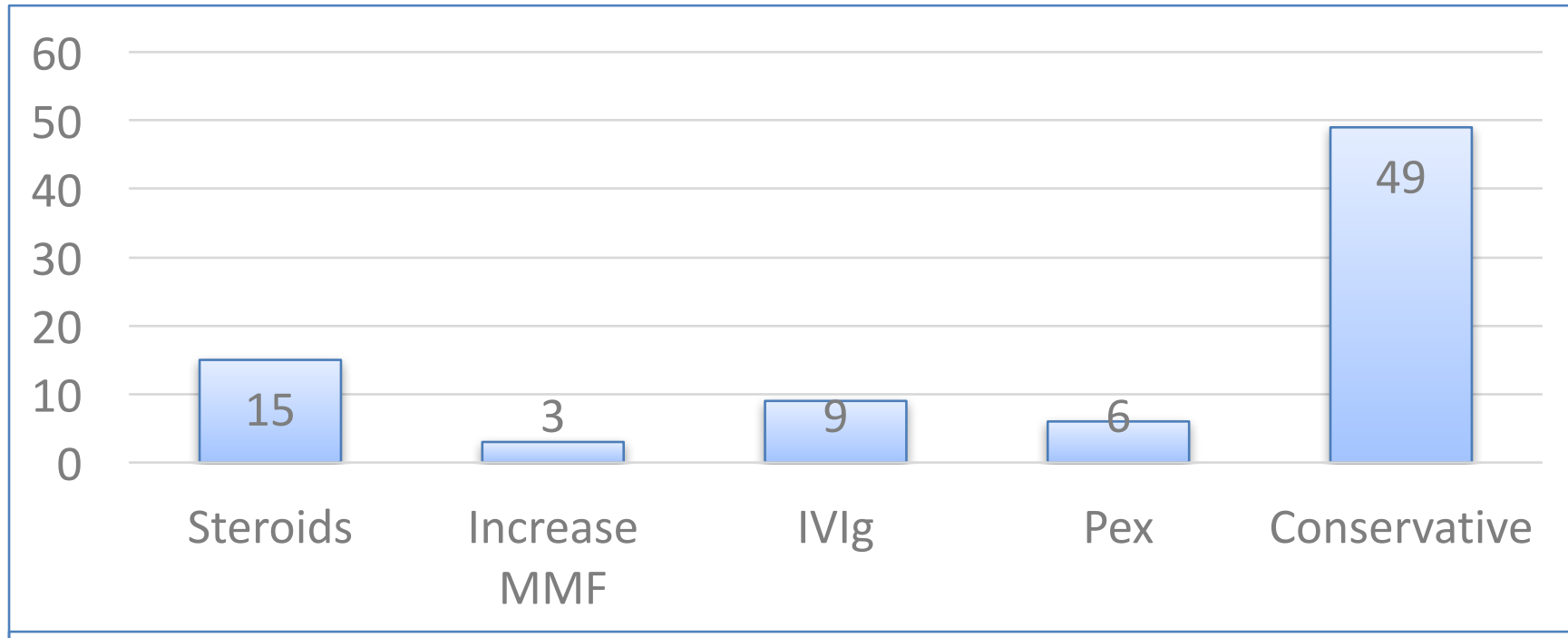

Figure 4. Management post biopsy. 4 patients with histological rejection were treated conservatively -2 of these had sepsis, the other 2 were mild with no perceived benefit from treatment. All remaining rejectors bar one (who received IVlg only) received methylprednisolone (MP) +/- increased MMF/IVIg/Pex. 2 patients with normal biopsies received PEx (for DSA); I patient with a normal biopsy received MP pre-biopsy.

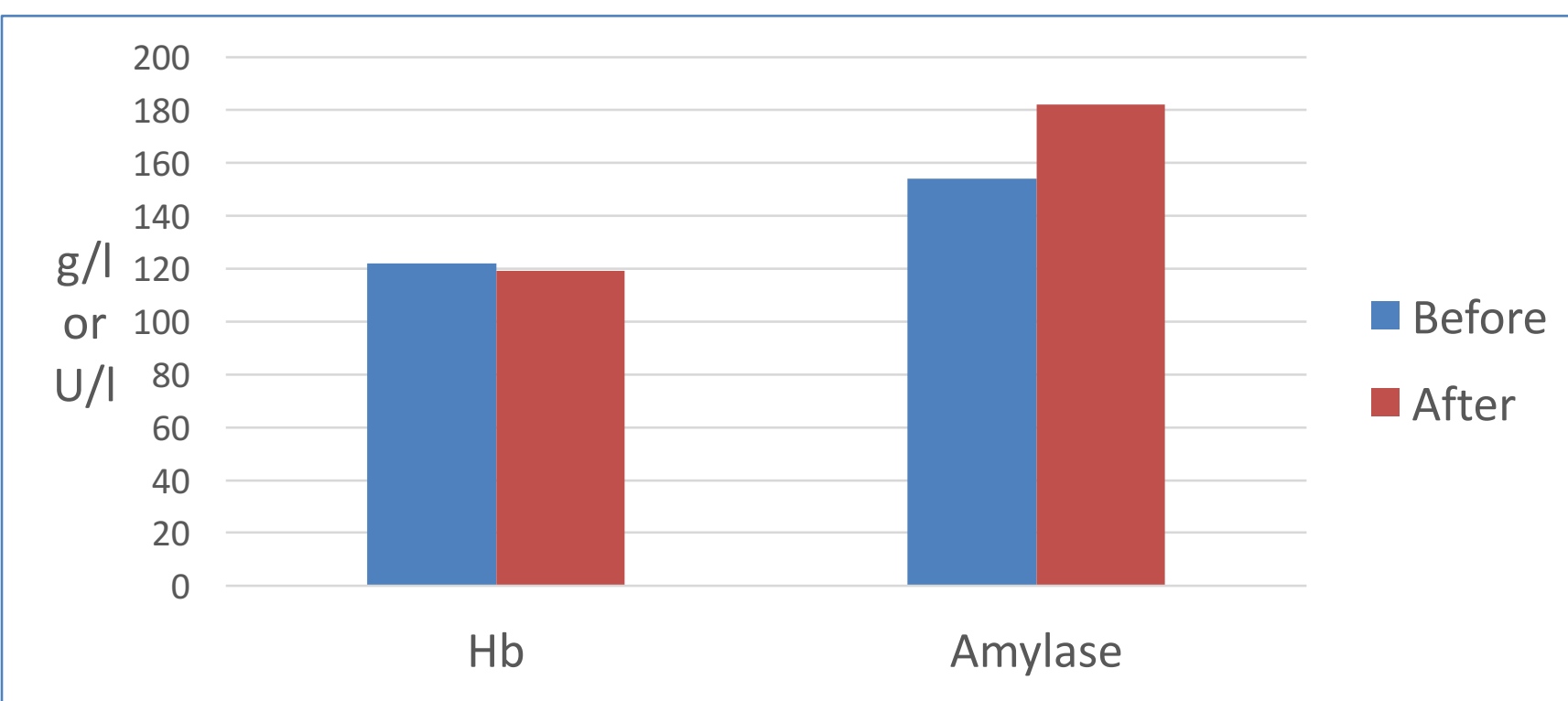

Figure 5. Change in haemoglobin and amylase following biopsy. One patient developed immediate post-biopsy haemorrhage requiring 4 units red cells and I unit FFP. 5 patients had $>50 \%$ rise in amylase post biopsy; I result was I029U/I and was treated as biopsy-induced acute pancreatitis.

\section{Conclusions}

- Ultrasound-guided allograft pancreas biopsy can be performed safely

- Adequate tissue for making a histological diagnosis can be obtained in the majority

- Unnecessary and/or empirical immunotherapy can be avoided, as was the case in $49 / 72(68 \%)$ of cases 